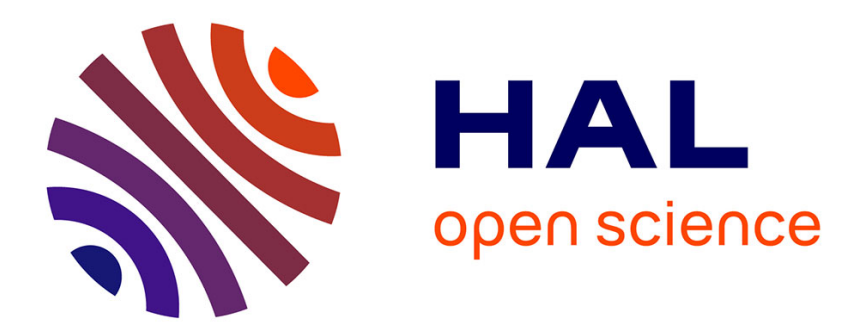

\title{
Electron localization function from density components
} Julien Pilmé

\section{To cite this version:}

Julien Pilmé. Electron localization function from density components. Journal of Computational Chemistry, 2017, 10.1002/jcc.24672 . hal-01398873

\section{HAL Id: hal-01398873 https: / hal.sorbonne-universite.fr/hal-01398873}

Submitted on 17 Nov 2016

HAL is a multi-disciplinary open access archive for the deposit and dissemination of scientific research documents, whether they are published or not. The documents may come from teaching and research institutions in France or abroad, or from public or private research centers.
L'archive ouverte pluridisciplinaire HAL, est destinée au dépôt et à la diffusion de documents scientifiques de niveau recherche, publiés ou non, émanant des établissements d'enseignement et de recherche français ou étrangers, des laboratoires publics ou privés. 


\title{
Electron Localization Function From Density Components
}

\author{
Julien Pilmé 1 \\ ${ }^{1}$ Sorbonne Universités, UPMC Univ Paris 06, CNRS, Laboratoire de Chimie Théorique \\ CC 137 - 4, place Jussieu F. 75252 PARIS CEDEX 05 - France \\ E-mail: pilme@lct.jussieu.fr
}

\begin{abstract}
This work addresses the decomposition of the Electron Localization Function (ELF) into partial density contributions using an appealing split of kinetic energy densities. Regarding the degree of the electron localization, the relationship between ELF and its usual spin-polarized formula is discussed. A new polarized ELF formula, built from any subsystems of the density, and a localization function, quantifying the measure of electron localization for only a subpart of the total system are introduced. The methodology appears tailored to describe the electron localization in bonding patterns of subsystems, such as the local nucleophilic character. Beyond these striking examples, this work opens up opportunities to describe any electronic properties that depend only on subparts of the density in atoms, molecules or solids.
\end{abstract}

\section{Introduction}

Over the two past decades, the analysis of the gradient field of the Electron Localization Function (ELF) has helped in understanding the concept of electron-pair localization in numerous molecules and solids. ${ }^{[1-4]}$ However, the long-running debate over critics of ELF about its definitions and its physical interpretations is still relevant. ${ }^{[5-12]}$ These include the original formulation of Becke and Edgecombe which lies on the conditional same-spin pair probability $D_{\sigma}$ scaled by the homogeneous electron gas (HEG) kinetic energy density $D_{\sigma}^{0}{ }^{[13]}$, both $D_{\alpha}$ and $D_{\beta}$ being separately computed. The kernel $\chi_{\sigma}$ of ELF is then expressed from $n_{\sigma}$ occupied molecular orbitals $\varphi_{i \sigma}$, as follows:

$$
\chi_{\sigma}=\frac{\mathrm{D}_{\sigma}}{\mathrm{D}_{\sigma}^{0}}=\frac{\sum_{\mathrm{i}}^{\mathrm{n}_{\sigma}}\left|\nabla \varphi_{\mathrm{i} \sigma}\right|^{2}-\frac{1}{4} \frac{\left|\nabla \rho_{\sigma}\right|^{2}}{\rho_{\sigma}}}{\mathrm{D}_{\sigma}^{0}}=\frac{\tau_{\sigma}-\frac{1}{8} \frac{\left|\nabla \rho_{\sigma}\right|^{2}}{\rho_{\sigma}}}{\mathrm{c}_{\mathrm{f}} 2^{2 / 3} \rho_{\sigma}^{5 / 3}}
$$

and $\mathrm{ELF}_{\sigma}=\frac{1}{1+\chi_{\sigma}^{2}}$

where $\rho_{\sigma}$ is the $\sigma$-spin density and $\tau_{\sigma}$ is the spin contribution to the positive definite kinetic energy density. ELF $\sigma$ ranges from 0 to 1 where 1 is a situation void of Pauli repulsion, i.e. a high probability of finding electron localization. Several theoretical studies have shown that the separated $\mathrm{ELF}_{\alpha / \beta}$ components are quite useful to evaluate the localization of unpaired electron in radicals. ${ }^{[14-16]}$ In a similar manner, $E E_{\sigma / \pi}$ components were conducted from $\rho_{\sigma} / \rho_{\pi}$ groups of molecular orbitals. ${ }^{[14,15,17,18]}$ These latter studies have been mainly motivated by the lack of assessment on the individual $\sigma / \pi$ bonding pictures in ELF based on the total electron density (see below). They have notably proved to be meaningful to quantify the concept of resonance and to rationalize the aromaticity of ring molecules. Note that $\mathrm{ELF}_{\sigma / \pi}$ components are in general isotopological to the termed ELI-D function which can be exactly decomposed as a sum of partial orbital contributions ${ }^{[19-21]}$. ELI-D is a variant form of the Electron Localizability Indicator (ELI), this latter being derived from the 
electron pair density without any reference to the uniform electron gas.

Turning to the original definition in terms of $\sigma$-spin density, a functional form of ELF based on the total electron density can be obtained for the closed-shell systems in which $\rho_{\alpha}$ $=\rho_{\beta}=\rho / 2$. In this latter case, the kernel of this total ELF becomes:

$$
\chi=\frac{\tau-\frac{1}{8} \frac{|\nabla \rho|^{2}}{\rho}}{\mathrm{c}_{\mathrm{f}} \rho^{5 / 3}} \text { and ELF }=\frac{1}{1+\chi^{2}}
$$

where $\tau$ is the positive definite kinetic energy density and $\rho$ is the total electron density. Note that equation (2) cannot be derived from any components of the total density, such as $\rho_{\sigma} / \rho_{\pi}$, and it should be strictly restricted to closed-shell systems. Actually, the equation (2) is also thoroughly used for open-shell systems because it can be obtained in a conceptually different way. Indeed, a physical interpretation of equation (2) was later attributed ${ }^{[22]}$ in terms of the local kinetic energy densities as:

$$
\chi=\frac{\tau^{\text {Pauli }}}{\tau^{\mathrm{h}}}=\frac{\tau-\tau^{\mathrm{w}}}{\tau^{\mathrm{h}}}=\frac{\tau-\frac{1}{8} \frac{|\nabla \rho|^{2}}{\rho}}{\mathrm{c}_{\mathrm{f}} \rho^{5 / 3}}
$$

Where $\tau^{\mathrm{w}}$ is the von Weizsäcker kinetic energy density, i.e. the kinetic energy of a density model system in which the antisymmetry is switched off. ${ }^{[23]}$ The kernel $\chi$ is now interpreted in terms of Pauli kinetic energy density $\tau^{\text {Pauli }}$, i.e. the local excess kinetic energy due to the Pauli exclusion principle. Note that some years later, ELF was also extended to $a b$ initio correlated wave functions ${ }^{[24]}$.

In its original definition (equation 1 ) as well as in its functional form (equation 3), ELF does not necessarily go to zero for vanishing density. Numerical instabilities can also appear in low density regions, typically when $D_{\sigma}$ tends to zero faster than $\rho$. To address these issues, Savin et al. have introduced in the equation (3), a shifting constant $\varepsilon=2.871 .10^{-5}$ to $\tau^{\text {Pauli }}$ quantity ${ }^{[25]}$. This compels ELF to be less than 0.5 when $\rho \leq 10^{-3}$ bohr $^{-3}$ and yields ELF to zero with the electron density.

The kinetic energy interpretation of the total ELF (equations 3) extends its validity of systems where $\tau^{\text {pauli }}$ can be properly evaluated within Density Functional Theory, the $\sigma$ dependence (equation 1) no longer appearing in the equations (2) and (3). Thus, details about bond pictures obtained from individual orbital contributions such as the $\sigma / \pi$ decomposition, are lost in the total ELF ${ }^{[17]}$. Actually, the way to decompose the function into any density components is unclear, the total ELF being neither the sum nor the average of its partial density contributions. However, in analogy to the spin-density functional theory ${ }^{[26]}$, a such decomposition in terms of spin densities (spinpolarized ELF) was proposed by Kohout and Savin ${ }^{[27]}$ as follows:

$$
\begin{gathered}
\chi_{S}=\frac{\tau_{\alpha}^{\text {Pauli }}+\tau_{\beta}^{\text {Pauli }}}{\tau_{\alpha}^{\mathrm{h}}+\tau_{\beta}^{\mathrm{h}}}=\frac{\tau-\frac{1}{8} \frac{\left|\nabla \rho_{\alpha}\right|^{2}}{\rho_{\alpha}}-\frac{1}{8} \frac{\left|\nabla \rho_{\beta}\right|^{2}}{\rho_{\beta}}}{2^{2 / 3} c_{\mathrm{f}}\left(\rho_{\alpha}{ }^{5 / 3}+\rho_{\beta}{ }^{5 / 3}\right)} \\
\text { and } \mathrm{ELF}_{\mathrm{s}}=\frac{1}{1+\chi_{\mathrm{s}}{ }^{2}}
\end{gathered}
$$

Note that $\mathrm{ELF}_{\mathrm{s}}$ is also computed with the shifting constant introduced by Savin et al ${ }^{[25]}$. Although the equation (4) addresses the challenge to decompose the total ELF in terms of spin densities, the relationship between equations (3) and (4) remains unclear because it is only possible once again to obtain $\chi_{s}=\chi$ in the closedshell systems. In open-shell systems, these equations generally differ.

The purpose of the present paper is to discuss the relationship between the total ELF and its partial density components. A particular focus on the relationship between the spinpolarized ELF $E_{s}$ (equation 4) and its usual functional form (equation 3 ) will be examined in terms of degree of the electron localization. Another purpose of the report is to explore how, from the total ELF, the electron localization can be evaluated in any subparts of the density. 


\section{ELF decomposition into density components}

The total electron density can be trivially decomposed into orbitals components. Under the only constraints of the single or double occupation $\varepsilon_{\mathrm{i}}$ for each spatial molecular orbital $\varphi_{i}$ (Hartree-Fock or Kohn-Sham formalisms), $\rho$ can be written as a function of subshell electron densities of $n_{1}$ and $n_{2}$ occupied MOs:

$$
\rho=\sum_{\mathrm{i}=1}^{\mathrm{n}} \varepsilon_{\mathrm{i}}\left|\phi_{\mathrm{i}}\right|^{2}=\sum_{\mathrm{i}=1}^{\mathrm{n}_{1}} \varepsilon_{\mathrm{i}}\left|\varphi_{\mathrm{i}}\right|^{2}+\sum_{\mathrm{i}=\mathrm{n}_{1}+1}^{\mathrm{n}_{2}} \varepsilon_{\mathrm{i}}\left|\varphi_{\mathrm{i}}\right|^{2}=\rho_{1}+\rho_{2}
$$

With $n_{1}+n_{2}=n$.

Note that this decomposition does not restricted to only spin densities, any split of the total density chemically significant could be considered.

A dimensionless normalized quantity $x$ can be expressed as follows:

$$
x(\mathbf{r})=\frac{\rho_{1}(\mathbf{r})}{\rho(\mathbf{r})} \text { or } 1-x(\mathbf{r})=\frac{\rho_{2}(\mathbf{r})}{\rho(\mathbf{r})}
$$

$x$ generally depends on the position $r$ because the ratios $\rho_{1} / \rho$ or $\rho_{2} / \rho$ do not remain constant over the molecular space and consequently $x$ changes from $1\left(\rho_{1}=\rho\right)$ to $0\left(\rho_{2}=\rho\right)$. Note that in this context, the kinetic energy density $\tau$ can also be trivially separated into two parts $\tau=\tau_{1}+\tau_{2}$ such as:

$$
\tau_{1}=\frac{1}{2} \sum_{\mathrm{i}=1}^{\mathrm{n}_{1}}\left|\nabla \phi_{\mathrm{i}}\right|^{2} \text { and } \tau_{2}=\frac{1}{2} \sum_{\mathrm{i}=\mathrm{n}_{1}+1}^{\mathrm{n}_{2}}\left|\nabla \varphi_{\mathrm{i}}\right|^{2}
$$

\section{The von Weizsäcker kinetic energy density}

Generally, $\tau^{\mathrm{w}}$ of the total system differs from the summation of kinetic energies for each subsystem $\tau_{1}{ }^{\mathrm{w}}+\tau_{2}{ }^{\mathrm{w}}$. Hence, the decomposition of $\tau^{\mathrm{w}}$ remains a tricky problem. The first step is to remark that $\tau^{\mathrm{w}}$ can be split in the following way $^{[28]}$ :

$$
\begin{aligned}
\tau^{\mathrm{w}}= & \frac{1}{8} \frac{\left|\nabla\left(\rho_{1}+\rho_{2}\right)\right|^{2}}{\rho_{1}+\rho_{2}} \\
& =\frac{\mathrm{x}}{8} \frac{\left|\nabla \rho_{1}\right|^{2}}{\rho_{1}}+\frac{(1-\mathrm{x})}{8} \frac{\left|\nabla \rho_{2}\right|^{2}}{\rho_{2}}+\frac{2 \nabla \rho_{1} \nabla \rho_{2}}{8\left(\rho_{1}+\rho_{2}\right)}
\end{aligned}
$$

Therefore, a term $\tau_{c}{ }^{w}$ where $\rho_{1}$ and $\rho_{2}$ are mixed, can be underlined as the difference between the summation $\tau_{1}{ }^{\mathrm{w}}+\tau_{2}{ }^{\mathrm{w}}$ and the total $\tau^{\mathrm{w}}$ :

$$
\begin{aligned}
\tau_{c}{ }^{w}= & \tau_{1}{ }^{w}+\tau_{2}{ }^{w}-\tau^{w} \\
& =\frac{1}{8} \frac{\left|\nabla \rho_{1}\right|^{2}}{\rho_{1}}(1-x)+\frac{1}{8} \frac{\left|\nabla \rho_{2}\right|^{2}}{\rho_{2}} x-\frac{2 \nabla \rho_{1} \nabla \rho_{2}}{8 \rho} \\
\tau_{c}{ }^{w} & =\frac{1}{8} \frac{\left(\rho_{1}+\rho_{2}\right)\left|(1-x) \nabla \rho_{1}-x \nabla \rho_{2}\right|^{2}}{\rho_{1} \rho_{2}} \geq 0
\end{aligned}
$$

Interestingly, the equation (8) underlines that $\tau_{c}{ }^{w}$ is a positive quantity which implies that $\tau^{\text {pauli }}>$ $\left(\tau_{1}^{\text {Pauli }}+\tau_{2}^{\text {Pauli }}\right)$ underlying a small overestimation of the electron localization if the summation $\tau_{1}{ }^{w}$ $+\tau_{2}{ }^{\mathrm{w}}$ is used instead of the total $\tau^{\mathrm{w}}$. This result is illustrated in Figure 1 with $\rho_{1}=\rho_{\alpha}$ and $\rho_{2}=\rho_{\beta}$. Here, the mixed term appears as negligible in the core regions because $\rho_{\alpha}$ and $\rho_{\beta}$ are equal ( $x$ becomes 0.5 ). In the valence regions, the loss of the mixed term becomes conspicuous (see Figure 1).

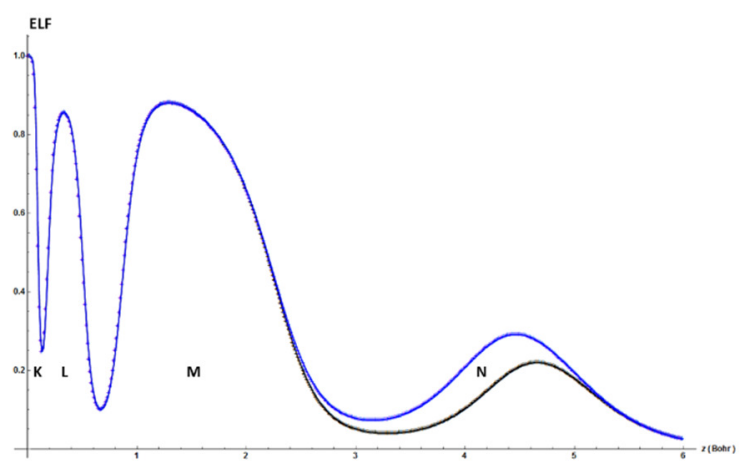

Figure 1: ELF values along the $z$ axis of the $K\left({ }^{2} S\right)$ atom calculated at the B3LYP /aug-cc-pVTZ level of theory. ELF (equation 3) is displayed in black while ELF without the mixed term $\tau_{c}{ }^{\mathrm{w}}$ is displayed in blue. The capital letters give the shells. 
Generally, if $x$ does not depend of $r$, namely that the ratio $\rho_{1} / \rho_{2}$ remains unchanged over the space, the equation (8) evidences that the mixed term is lost $\left(\tau_{c}{ }^{\mathrm{w}}=0\right)$ since $\left|\nabla \rho_{1}\right|=x|\nabla \rho|$ and $\left|\nabla \rho_{2}\right|=(1-x)|\nabla \rho|$, and thereby simplifying $\tau^{\mathrm{w}}$ as the sum $\tau_{1}{ }^{\mathrm{W}}+\tau_{2}{ }^{\mathrm{W}}$. Note that applied to the spin-densities, this mixed term becomes zero for the closed-shell systems in which $x=1 / 2\left(\rho_{\alpha}=\rho_{\beta}=\right.$ $\rho / 2$ ) everywhere. It is worth noting that in practice, for most systems, $\tau_{c}{ }^{w}$ remains generally very small with respect to kinetic energy densities because the subsystem densities are mainly distributed in different regions of the molecular space.

\section{The HEG kinetic energy density}

The decomposition of $\tau^{\text {h }}$ into density components is not a trivial problem because $\tau^{\mathrm{h}}$ differs from the summation of kinetic energies for each subsystem. However, it has been previously shown that $\tau^{\text {h }}$ can be easily split as follows ${ }^{[28]}$ :

$$
\begin{aligned}
\tau^{h} & =c_{f} \rho^{5 / 3} \\
& =c_{f} x^{5 / 3} \rho^{5 / 3}+c_{f}\left(1-x^{5 / 3}\right) \rho^{5 / 3}=c_{f}\left(\rho_{1}^{5 / 3}+g(x) \rho_{2}{ }^{5 / 3}\right)
\end{aligned}
$$

where $g(x)=\frac{1-x^{5 / 3}}{(1-x)^{5 / 3}}$. The plot of $g(x)$ is displayed in Figure 2.

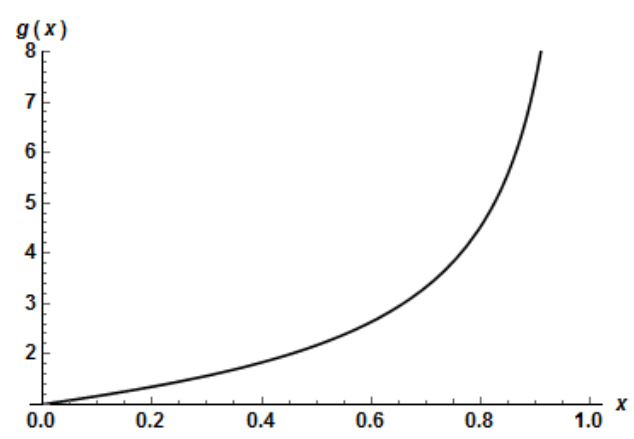

Figure 2: Plot of the $g(x)$ function

As shown in Figure 2, $g(0)$ is 1 and $g(x)$ goes to infinity while $x$ becomes 1 . Actually, at the limit $x \rightarrow 1$, the quantity $g(x) \rho_{2}^{5 / 3}$ occuring in the equation (9) switch to 0 while $x$ becomes 1 because $\rho_{2}$ becomes always zero faster than $g(x)$ goes to infinity. In a similar manner to the von Weizsäcker quantity, a term $\tau_{c}{ }^{h}$ where $\rho_{1}$ and $\rho_{2}$ are mixed, can be underlined as the difference between the summation $\tau_{1}{ }^{\mathrm{h}}+\tau_{2}{ }^{\mathrm{h}}$ and the total $\tau^{\mathrm{h}}$ as follows:

$$
\begin{aligned}
\tau_{c}^{h} & =\tau_{1}^{h}+\tau_{2}^{h}-\tau^{h}=2^{2 / 3} c_{f} \rho_{1}^{5 / 3}+2^{2 / 3} c_{f} \rho_{2}^{5 / 3}-\tau^{h} \\
& =c_{f}\left(2^{2 / 3}-1\right) \rho_{1}^{5 / 3}+c_{f}\left(2^{2 / 3}-g(x)\right) \rho_{2}{ }^{5 / 3} \geq 0
\end{aligned}
$$

$\tau_{c}{ }^{\mathrm{h}}$ appears always positive even when $\mathrm{x}$ goes to $1\left(\rho_{1} \approx \rho, g(x) \rho_{2}{ }^{5 / 3} \rightarrow 0\right)$. It becomes only zero when $x=1 / 2$. Thus, the equation (10) shows that $\tau^{\mathrm{h}}<\left(\tau_{1}{ }^{\mathrm{h}}+\tau_{2}{ }^{\mathrm{h}}\right)$ underlying an overestimation of the electron localization if the summation $\tau_{1}{ }^{\mathrm{h}}+$ $\tau_{2}{ }^{h}$ is used instead of the total $\tau^{\mathrm{h}}$. Once again, this result is shown in Figure 3 where $\rho_{1}=\rho_{\alpha}$ and $\rho_{2}=$ $\rho_{\beta}$. Note that the mixed term appears quite negligible in the core regions where $x=1 / 2$ since $\rho_{\alpha}=\rho_{\beta}$.

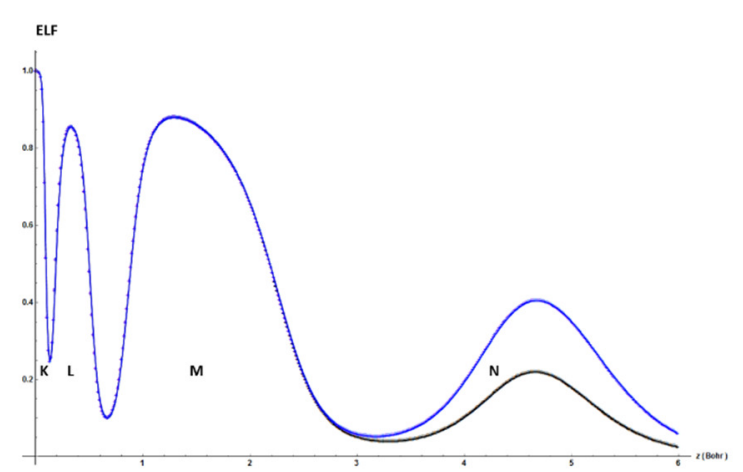

Figure 3: ELF values along the $z$ axis of the $\mathrm{K}\left({ }^{2} \mathrm{~S}\right)$ atom calculated at the B3LYP /aug-cc-pVTZ level of theory. ELF (equation 3 ) is displayed in black while ELF without the mixed term $\tau_{c}{ }^{h}$ is displayed in blue. The capital letters give the shells.

\section{The polarized ELF}

At this stage, one can easily grasp how density components play such a role in determining the degree of the electron localization measured by the total ELF (equation 3). Indeed, the kernel of ELF can be now related to its kinetic energy components: 
$\chi=\frac{\tau_{1}^{\text {Pauli }}+\tau_{2}^{\text {Pauli }}+\tau_{c}^{w}}{\tau_{1}^{h}+\tau_{2}^{h}-\tau_{c}^{h}} \geq \frac{\tau_{1}^{\text {Pauli }}+\tau_{2}^{\text {Pauli }}}{\tau_{1}^{h}+\tau_{2}^{h}}$

The equation (11) is a decomposition of ELF into unmixed $\left(\tau_{1}{ }^{\text {Pauli }}, \tau_{2}{ }^{\text {Pauli }}, \tau_{1}{ }^{\mathrm{h}}, \tau_{2}{ }^{\mathrm{h}}\right)$ and mixed $\left(\tau_{\mathrm{c}}{ }^{\mathrm{w}}\right.$, $\left.\tau_{c}{ }^{h}\right)$ terms. It highlights that if both mixed terms are omitted, the degree of electron localization is overestimated. Applied to spin-densities $\left(\rho_{1}=\right.$ $\left.\rho_{\alpha}, \rho_{2}=\rho_{\beta}\right)$, the spin-polarized $\chi_{s}$ formula (equation 4 ) can be directly obtained from $\chi$ if these both mixed terms are dismissed. Nevertheless, the equation (11) shows that in the case of closed-shell $\left(x=1 / 2, \rho_{\alpha}=\rho_{\beta}=\rho / 2\right)$, the relation $\chi^{s}=\chi$ remains exactly verified since the mixed terms become exactly zero over the space. Beyond the connection between $\chi^{s}$ and $\chi$, a polarized form of ELF taking account mixed terms, even approximatively, should be able to properly describe the degree of localization in the whole molecular space. Thus, a polarized ELF as close as possible to ELF $_{\mathrm{s}}$, could be expressed as a sum of each Pauli kinetic energy weighted by $x$ components where the HEG reference occuring in $\mathrm{ELF}_{\mathrm{s}}$ remains unchanged:

$$
\begin{aligned}
& \chi_{p}=\frac{2\left(x \tau_{1}^{\text {Pauli }}+(1-x) \tau_{2}^{\text {Pauli }}\right)+\varepsilon}{\tau_{1}^{h}+\tau_{2}^{h}} \\
& =\frac{2\left(x \tau_{1}^{\text {pauli }}+(1-x) \tau_{2}^{\text {Pauli }}\right)+\varepsilon}{c_{f} 2^{2 / 3}\left(\rho_{1}^{5 / 3}+\rho_{2}^{5 / 3}\right)}
\end{aligned}
$$

$\operatorname{ELF}_{p}=\left(1+\chi_{p}{ }^{2}\right)^{-1}, \varepsilon$ being a shifting constant which constrains ELF to be less than 0.5 when $\rho \leq 10^{-}$ ${ }^{3}$ density. This constant was introduced for ELF (see the introduction section) in which $\varepsilon=$ 2.871.10-5. ${ }^{[25]}$ In the case of ELF,$\varepsilon=4.5574 .10^{-5}$ $\left[x^{5 / 3}+(1-x)^{5 / 3}\right]$. ELF and $E L F F_{p}$ go necessarily to zero for vanishing density (regions with numerical instabilities) whereas in its original definition (equations 1 and 3), ELF tends to 1 in low density regions. The factor 2 in the equation (12) holds the closed-shell connection since if $x=1 / 2, \chi_{p}$ is exactly equal to $\chi$ over the space. If $\mathrm{x}=0$ or $\mathrm{x}=1$, $\chi_{p}$ becomes proportional (factor 2) to the original expression of Becke and Edgecombe (equation 1).
Three examples of comparative behaviors of ELF,$E L F_{s}$ and the ELF (equation 3) are displayed in Figure 4. We can notice that both $E F_{p}$ and ELF display very similar profiles, $E L F_{p}$ appearing almost isotopological to ELF since the location and properties of critical points (local minima and maxima) are almost the same in the whole molecular space.

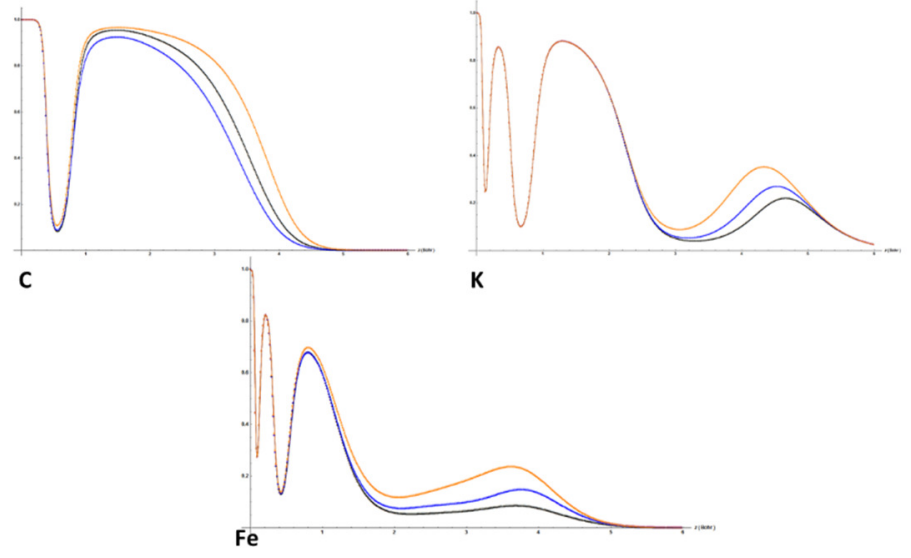

Figure 4: ELF values along the $\mathrm{z}$ axis of $\mathrm{C}\left({ }^{3} \mathrm{P}\right), \mathrm{K}\left({ }^{2} \mathrm{~S}\right)$ and $\mathrm{Fe}\left({ }^{5} \mathrm{D}\right)$ atoms calculated at the B3LYP /aug-cCpVTZ level of theory. ELF (equation 3 ), ELF $F_{p}$ (equation 12 with $\rho_{1}=\rho_{\alpha}$ and $\rho_{2}=\rho_{\beta}$ ) and ELF (equation 4 ) values are respectively displayed in black, blue and orange.

However, as regards the spin-polarized $E_{L} F_{s}$ (equation 4), the function can slightly differ from ELF in valence regions where the single electron character dominates. As discussed above, this is due to the overestimation of the degree of localization in $\mathrm{ELF}_{\mathrm{s}}$.

The distance between two minima of the considered function (ELF, ELF $\mathrm{p}$ or $\mathrm{ELF}_{\mathrm{s}}$ ) can be defined as a shell radius and charts the frontier between the spherical basin centered on the maxima location. The shell population can be then computed by the integration of the density over the basin's volume. For closed-shell systems, radii and populations of three ELF, ELF and $E L F_{s}$ are obviously the same (closed-shell connection). For open-shell systems, small differences are observed using the three formulas. The radius and the population of inner shells ( $K$ and $L$ ) have the same magnitude whatever the considered function. Conversely, the radius and the population of outer shells ( $M$ 
and $\mathrm{N}$ ) differ depending on the considered function. By way of example, consider the potassium atom $\mathrm{K}\left({ }^{2} \mathrm{~S}\right)$. The radius of the $\mathrm{M}$ shell decreases from 3.28 bohr for ELF to 3.06 bohr for $E L F_{s}$. A radius of 3.20 bohr is found for ELF $F_{p}$ which is close to that of ELF. Similar behavior is observed for the populations. The population of the $\mathrm{M}$ shell decreases from 8.05 e for ELF to 7.94 $e$ for $E_{L F}$. This population is 8.02 e for $\mathrm{ELF}_{\mathrm{p}}$ which is close to that of ELF. Thus, these results show that $E L F_{p}$ describes more adequately the degree of electron localization than $\mathrm{ELF}_{\mathrm{s}}$ where mixed terms are dismissed.

\section{Electron localization from density subsystems}

As previously discussed by some authors ${ }^{[15,17]}$, the total ELF (equations 3 ) lacks of assessment on the individual bonding pictures such as the conventional $\sigma / \pi$ scheme. Of course, one could use separated ELF components (for example $E L F_{\alpha / \beta}$ or $E L F_{\sigma / \pi}$ ) to measure the localization in subparts of the total density. However, as seen from the above discussion, the ELF components cannot be, in general, properly connected to the total ELF without including mixed terms. In addition, the ELF component of a single orbital has no physical meaning (ELF becomes 1 in the whole space). In this case, ELF decays only due to the shifting constant introduced by Savin et al. Overall, a function $\chi_{x}$ quantifying a meaningful measure of electron localization for only a subpart of the total density system (for example $\rho_{1}$ ) needs to answer to following properties:

1. The localization is inversely proportional to the number of electrons delocalized in the subsystem, i.e. if $x \rightarrow 0$, the function $\chi_{x}$ goes to infinity and then $1 /\left(1+\chi_{x}^{2}\right)$ goes to zero.

2. The closed-shell connection need to be sustained, i.e. $\chi_{x}=\chi$ when $x=1 / 2$.

These two criteria are fulfilled with the following equation:

$$
\chi_{x}=\frac{\chi}{2 x} \text { and } \mathrm{ELF}_{\mathrm{x}}=\frac{1}{1+\chi_{x}^{2}}
$$

Indeed, ELF $x$ becomes zero when $x \rightarrow 0$. The factor 2 holds the closed-shell connection since if $\mathrm{x}=1 / 2, \mathrm{ELF}_{\mathrm{x}}=\mathrm{ELF}$. Note that $\mathrm{ELF}_{\mathrm{x}}$ is also computed with the shifting constant introduced by Savin et $\mathrm{al}^{[25]}$. It is worth noting that if the subsystem involves only a single molecular orbital $\varphi$, i.e. $\rho_{1}=|\varphi|^{2}, \chi_{x}$ differs from zero while $\tau_{1}{ }^{\text {pauli }}$ becomes zero over the molecular space ${ }^{[8]}$.

The calculations were performed on a set of prototype systems where the separation of group of molecular orbitals within their symmetry, is chemically intuitive. Figure 5 depicts the electron localization domains computed with ELF $_{\mathrm{x}}$ for selected orbital dissections: core/valence separation in the benzene $\left(\rho=\rho_{c}+\rho_{v}\right), \sigma / \pi$ separation in the benzene $\left(\rho=\rho_{\sigma}+\rho_{\pi}\right)$, separation of spindensities in the radical $\mathrm{C}_{6} \mathrm{H}_{4}\left(\rho=\rho_{\alpha}+\rho_{\beta}\right)$.

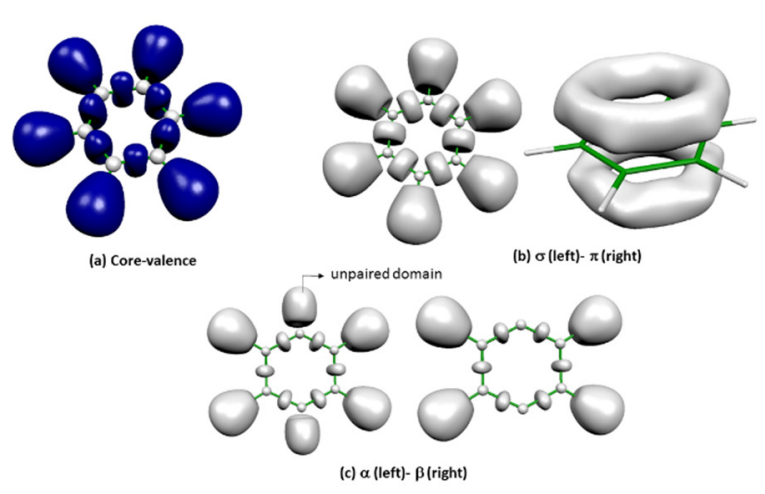

Figure 5. ELF $\mathrm{F}_{\mathrm{x}}$ localization domains ( $E L F=0.85$ except for $E L F_{x, \pi}=0.6$ ). (a) Separation into core and valence regions of the benzene molecule. Light grey: $\rho_{1}=\rho_{\text {core }}$ and blue: $\rho_{1}=\rho_{\text {valence. }}$ (b) Separation into $\sigma$ and $\pi$ regions of the benzene molecule. Left: : $\rho_{1}=\rho_{\sigma}$ and right : $\rho_{1}=\rho_{\pi}$ (c) Separation of density-spin components of the $\mathrm{C}_{6} \mathrm{H}_{4}$ molecule. Left: $\rho_{1}=\rho_{\alpha}$ and right: $\rho_{1}=\rho_{\beta}$.

One can see that in all cases, the ELF $F_{x}$ topologies match closely with the chemically meaningful pictures. By way of example, consider the $\alpha$ and $\beta$ localization domains in the Phenyl radical molecule. We observe, in Figure 5, an agreement between the topological domains displayed by $E \mathrm{LF}_{\mathrm{X}}$ and those of $E L F_{\alpha / \beta}$ components. They 
clearly show the unpaired domains of $\alpha$ functions while these domains do not exist in $\beta$ functions.

We now turn to the $\pi$ localization domains in the benzene displayed in Figures 5 and 6.
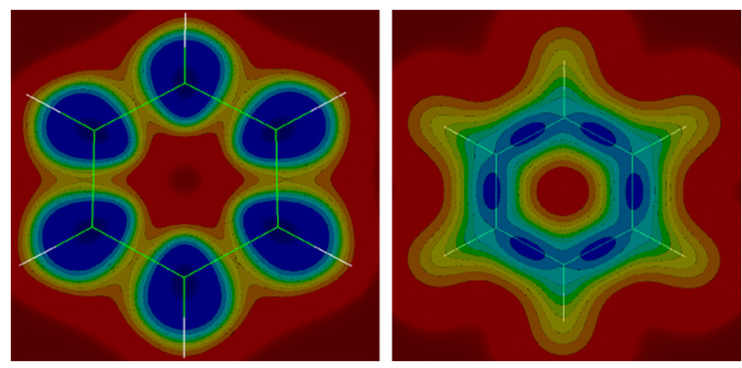

Figure 6. Compared localization domains of $\mathrm{ELF}_{\pi}$ (left) and $\mathrm{ELF}_{\mathrm{x}, \pi}$ (right) depicted in the molecular plane of the benzene molecule. Blue regions are domains of high electron localization while red regions are domains of low electron localization.

Regarding the topology of ELF $F_{x, \pi}$, it corresponds to a typically delocalized double bond picture where the attractors (local maxima) of bonding basins $\mathrm{C}-\mathrm{C}$ are located between the carbon atoms rather than on the carbon atoms. Note that this statement drawn from ELF $F_{x}$ differs from the topology of the single $\mathrm{ELF}_{\pi}$ component (see Figure 6) where the localization domains of C-C bonds are located on the carbon atoms above and below the molecular plane.

Further example of ELF $F_{x}$ can be gained in a context of frontier reactivity theory ${ }^{[26,29,30]}$ with $\rho_{1}=\varepsilon_{\text {номо }}\left|\varphi_{\text {номо }}\right|^{2}$ and $x=\varepsilon_{\text {номо }}\left|\varphi_{\text {номо }}\right|^{2} / \rho$. The ELF $F_{x}$ topology is then expected to simply stands for the contribution of the frontier orbital to the pair regions in the studied systems. Within a frozen orbital approximation, i.e. orbitals remaining unchanged upon addition and subtraction of one electron, $\rho_{1}$ is simply reduces to the Fukui function $\mathrm{f}^{-}$which is a local descriptor of the nucleophilicity. ${ }^{[31-34]}$ Overall, Fukui functions are powerful tools to get an insight into the reactivity since they allow to select the electrophilic and nucleophilic regions of the molecular space.

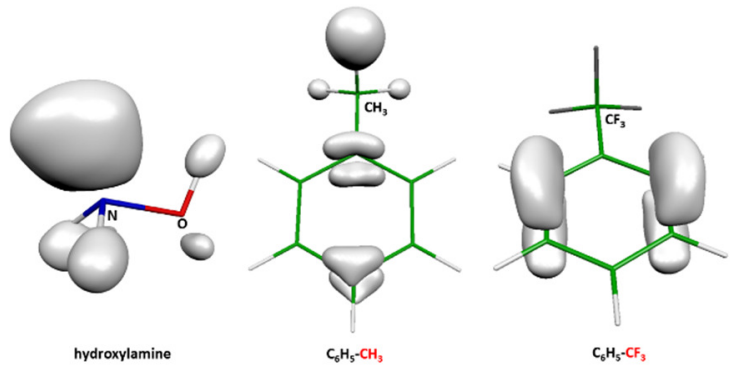

Figure 7. ELF $F_{x}$ localization domains built from the single Highest Occupied Molecular Orbital $\left(\rho_{1}=2\right.$ $\left|\varphi_{\text {номо }}\right|^{2}$ ) for $\mathrm{NH}_{2} \mathrm{OH}(\mathrm{ELF}=0.6)$, Toluene ( $\left.\mathrm{ELF}=0.75\right)$ and Trifluorotoluene ( $E L F=0.75)$ molecules.

The electron localization domains of $\mathrm{ELF}_{\mathrm{x}}$ for three selected systems are reported in Figure 7. For the $\mathrm{NH}_{2} \mathrm{OH}$ molecule, the ELF $\mathrm{F}_{\mathrm{x}}$ topology yields non-bonding basins accounting for nitrogen and oxygen lone pairs, no $\mathrm{N}-\mathrm{O}$ bonding oxygen basin was found. This can be conveniently interpreted by means of a bifurcation theory where the value of isosurfaces when basins begin to split (points of bifucation) are related to a measure of the electron localization and to the chemical interaction among the different basins. ${ }^{[25]}$ Interestingly, the nitrogen and oxygen non-bonding basins presents a weak ELF bifurcation value of 0.66 , thereby leaving a single nitrogen localization domain above the bifurcation value. In addition, the integration of the HOMO density $\int 2\left|\varphi_{\text {HOMO }}\right|^{2} \mathrm{dr}$ was computed over the basin volumes (populations) for all the basins of $\mathrm{NH}_{2} \mathrm{OH}$. A population of 0.31 e was found for the oxygen non-bonding basin whereas a population of 1.26 e was found for the nitrogen nonbonding basin. A population close to 0.1 e was found for each hydrogen basin. Hence, it is clear from these findings, that nitrogen center is predicted to be more nucleophilic than those corresponding to the oxygen and the hydrogen centers. This outcome is consistent with the well-known reactivity of the hydroxylamine predicts by Fukui analyses ${ }^{[35,36]}$. This is also experimental evidenced that the nitrogen atom is the most reactive, at least, for the protonation process. $^{[37]}$

The localization domains of $E F_{x}$ of $\mathrm{C}_{6} \mathrm{H}_{5} \mathrm{CH}_{3}$ (ortho-para reactivity) and $\mathrm{C}_{6} \mathrm{H}_{5} \mathrm{CF}_{3}$ 
(meta reactivity) are also displayed in Figure 7. Once again, excluding the ipso position of $\mathrm{C}_{6} \mathrm{H}_{5} \mathrm{CH}_{3}{ }^{[20]}$, the topology of ELF basins matches closely with the known local nucleophilicity of these compounds, notably predicts by the Fukui and the molecular orbital analyses ${ }^{[35]}$. Indeed, only localized valence basins are observed around carbons ortho and para for $\mathrm{C}_{6} \mathrm{H}_{5} \mathrm{CH}_{3}$ while only basins are displayed around carbons meta for $\mathrm{C}_{6} \mathrm{H}_{5} \mathrm{CF}_{3}$.

All Geometry optimizations were performed using the B3LYP hybrid functional ${ }^{[38]}$ combined with the aug-cc-pVTZ basis set for all calculations. The DFT calculations have been performed using the GAUSSIAN09 program package. ${ }^{[39]}$ Afterward, ELF calculations were carried out using a modified version of the TopMod and TopChem programs. ELF isosurfaces were drawn using the Molekel software. ${ }^{[40,41]}$

\section{Concluding Remarks}

In summary, this work has introduced a decomposition of ELF obtained from an appealing scheme of kinetic energy components. The relationship between ELF and its usual spinpolarized formula, proposed by Kohout and Savin a few years ago, has been clarified by showing how the spin-polarized ELF can be obtained if mixed terms are omitted. Beyond spin-densities, a new ELF polarized formula was proposed. Regarding the degree of the electron localization measured in ELF, a localization function quantifying the measure of electron localization for only a subpart of the total system was introduced. The function could be useful to evaluate electronic properties which depend only on subparts of the density (aromatic character, radicals...) in various chemical systems and notably to be useful for exploring the local chemical reactivity of donor systems.

\section{References}

[1] B. Silvi, R. J. Gillespie, C. Gatti. In Comprehensive Inorganic Chemistry II (Second Edition); Poeppelmeier, K., Ed.; Elsevier: Amsterdam, 2013, p 187-226.

[2] B. Silvi, J. Pilme, F. Fuster, M. E. Alikhani. In Metal-Ligand Interactions; Russo, N., Salahub, D.,Witko, M., Eds.; Springer Netherlands, 2003, p 241-284.

[3] T. Zhang, W. Lei, P. Liu, J. A. Rodriguez, J. Yu, Y. Qi, G. Liu, M. Liu. J Phys Chem C 2016, 120(5), 2777-2786.

[4] L. A. Mancera, D. M. Benoit. J Phys Chem A 2015, 119(12), 3075-3088.

[5] M. Rahm. J Chem Theory Comput 2015, 11(8), 3617-3628.

[6] P. L. Ayers, R. J. Boyd, P. Bultinck, M. Caffarel, R. Carbó-Dorca, M. Causá, J. Cioslowski, J. Contreras-Garcia, D. L. Cooper, P. Coppens, C. Gatti, S. Grabowsky, P. Lazzeretti, P. Macchi, Á. Martín Pendás, P. L. A. Popelier, K. Ruedenberg, H. Rzepa, A. Savin, A. Sax, W. H. E. Schwarz, S. Shahbazian, B. Silvi, M. Solà, V. Tsirelson. Comput Theor Chem 2015, 1053, 2-16.

[7] A. Savin. Journal of Molecular Structure: THEOCHEM 2005, 727(1-3), 127-131.

[8] J. K. Burdett, T. A. McCormick. J Phys Chem A 1998, 102(31), 6366-6372.

[9] M. Kohout, R. F. Wagner, Y. Grin. Theor Chem Acc 2002, 108(3), 150-156.

[10] M. Kohout, A. Savin. J Comput Chem 1997, 18(12), 1431-1439.

[11] M. Amaouch, E. Renault, G. Montavon, N. Galland, J. Pilmé. In Applications of Topological Methods in Molecular Chemistry; Chauvin, R., Lepetit, C., Silvi, B.,Alikhani, E., Eds.; Springer International Publishing: Cham, 2016, p 553-582.

[12] J. Pilmé, E. Renault, T. Ayed, G. Montavon, N. Galland. J Chem Theory Comput 2012, 8(9), 2985-2990.

[13] A. D. Becke, K. E. Edgecombe. J Chem Phys 1990, 92(9), 5397-5403.

[14] P. Fuentealba, E. Chamorro, J. C. Santos. In Theoretical and Computational Chemistry; Alejandro, T.-L., Ed.; Elsevier, 2007, p 57-85.

[15] J. C. Santos, W. Tiznado, R. Contreras, P. Fuentealba. J Chem Phys 2004, 120(4), 16701673. 
[16] J. Melin, P. Fuentealba. Int J Quantum Chem 2003, 92(4), 381-390.

[17] S. N. Steinmann, Y. Mo, C. Corminboeuf. Phys Chem Chem Phys 2011, 13(46), 2058420592.

[18] J. C. Santos, J. Andres, A. Aizman, P. Fuentealba. J Chem Theory Comput 2005, 1(1), 83-86.

[19] M. Kohout. Int J Quantum Chem 2004, 97(1), 651-658.

[20] M. Kohout, F. R. Wagner, Y. Grin. Int J Quantum Chem 2006, 106(7), 1499-1507.

[21] F. R. Wagner, V. Bezugly, M. Kohout, Y. Grin. Chem Eur J 2007, 13(20), 5724-5741.

[22] A. Savin, O. Jepsen, J. Flad, O. K. Andersen, H. Preuss, H. G. von Schnering. Angew Chem Int 1992, 31(2), 187-188.

[23] C. Von Weizsacker. Zeitschrift für Physik 1935, 96(7), 431-458.

[24] E. Matito, B. Silvi, M. Duran, M. Solà. J Chem Phys 2006, 125(2), 024301.

[25] A. Savin, B. Silvi, F. Colonna. Can J Chem 1996, 74(6), 1088-1096.

[26] R. Parr, W. Yang. Density-Functional Theory of Atoms and Molecules; Oxford University Press: New York, 1994.

[27] M. Kohout, A. Savin. Int J Quantum Chem 1996, 60(4), 875-882.

[28] M. S. Miao. J Phys A: Math Gen 2001, 34(39), 8171.

[29] P. Geerlings, F. De Proft, W. Langenaeker. Chem Rev 2003, 103(5), 17931874.

[30] E. Chamorro, J. Melin. Journal of Molecular Modeling 2015, 21(3), 1-3.

[31] K. Fukui. In Orientation and Stereoselection; Springer Berlin Heidelberg: Berlin, Heidelberg, 1970, p 1-85.

[32] R. G. Parr, W. Yang. J Am Chem Soc 1984, 106(14), 4049-4050.

[33] W. Yang, R. G. Parr. Proceedings of the National Academy of Sciences 1985, 82(20), 6723-6726.

[34] P. Fuentealba, C. Cardenas, R. Pino-Rios, W. Tiznado. In Applications of Topological
Methods in Molecular Chemistry; Chauvin, R., Lepetit, C., Silvi, B.,Alikhani, E., Eds.; Springer International Publishing: Cham, 2016, p 227-241. [35] P. Fuentealba, E. Florez, W. Tiznado. J Chem Theory Comput 2010, 6(5), 1470-1478.

[36] E. Chamorro, M. Duque, C. Cárdenas, J. Santos, W. Tiznado, P. Fuentealba. J Chem Sci (Bangalore, India) 2005, 117(5), 419-424.

[37] F. Angelelli, M. Aschi, F. Cacace, F. Pepi, G. de Petris. J Phys Chem 1995, 99(17), 65516556.

[38] P. J. Stephens, F. J. Devlin, C. F. Chabalowski, M. J. Frisch. J Phys Chem 1994, 98(45), 11623-11627.

[39] M. J. Frisch, G. W. Trucks, H. B. Schlegel, G. E. Scuseria, M. A. Robb, J. R. Cheeseman, G. Scalmani, V. Barone, B. Mennucci, G. A. Petersson, H. Nakatsuji, M. Caricato, X. Li, H. P. Hratchian, A. F. Izmaylov, J. Bloino, G. Zheng, J. L. Sonnenberg, M. Hada, M. Ehara, K. Toyota, R. Fukuda, J. Hasegawa, M. Ishida, T. Nakajima, Y. Honda, O. Kitao, H. Nakai, T. Vreven, J. A. Montgomery Jr., J. E. Peralta, F. Ogliaro, M. J. Bearpark, J. Heyd, E. N. Brothers, K. N. Kudin, V. N. Staroverov, R. Kobayashi, J. Normand, K. Raghavachari, A. P. Rendell, J. C. Burant, S. S. lyengar, J. Tomasi, M. Cossi, N. Rega, N. J. Millam, M. Klene, J. E. Knox, J. B. Cross, V. Bakken, C. Adamo, J. Jaramillo, R. Gomperts, R. E. Stratmann, O. Yazyev, A. J. Austin, R. Cammi, C. Pomelli, J. W. Ochterski, R. L. Martin, K. Morokuma, V. G. Zakrzewski, G. A. Voth, P. Salvador, J. J. Dannenberg, S. Dapprich, A. D. Daniels, Ö. Farkas, J. B. Foresman, J. V. Ortiz, J. Cioslowski, D. J. Fox. Gaussian, Inc.: Wallingford, CT, USA, 2009.

[40] P. Flukiger, H. P. Luthi, S. Portmann, J. Weber. Swiss Center for Scientific Computing: Manno, Switzerland, 2002.

[41] D. Kozlowski, J. Pilmé. J Comput Chem 2011, 32(15), 3207-3217. 
